\title{
A flexible mathematical model for matching of 3D surfaces and attributes
}

\section{Report}

\section{Author(s):}

Grün, Armin; Akca, Devrim

Publication date:

2005

Permanent link:

https://doi.org/10.3929/ethz-a-004908531

Rights / license:

In Copyright - Non-Commercial Use Permitted 


\title{
A flexible mathematical model for matching of 3D surfaces and attributes
}

\author{
Devrim Akca, Armin Gruen \\ Chair of Photogrammetry and Remote Sensing, Swiss Federal Institute of Technology (ETH) Zurich \\ ETH Hoenggerberg, CH-8093 Zurich, Switzerland, E-mail: <akca><agruen>@geod.baug.ethz.ch
}

\begin{abstract}
An algorithm for the least squares matching of overlapping 3D surfaces is presented. It estimates the transformation parameters between two or more fully 3D surfaces, using the Generalized Gauss-Markoff model, minimizing the sum of squares of the Euclidean distances between the surfaces. This formulation gives the opportunity of matching arbitrarily oriented 3D surfaces simultaneously, without using explicit tie points. Besides the mathematical model and execution aspects we give further extension of the basic model. The first extension is the simultaneous matching of sub-surface patches, which are selected in cooperative surface areas. It provides a computationally effective solution, since it matches only relevant multi-subpatches rather than the whole overlapping areas. The second extension is the matching of surface geometry and its attribute information, e.g. reflectance, color, temperature, etc., under a combined estimation model. We give practical examples for the demonstration of the basic method and the extensions.
\end{abstract}

Keywords: Least squares 3D surface matching, point clouds, registration, laser scanning, intensity matching

\section{INTRODUCTION}

For 3D object modeling data acquisition must be performed from different standpoints. The derived local point clouds must be transformed into a common system. This procedure is usually referred to as registration. In the past, several efforts have been made concerning the registration of $3 \mathrm{D}$ point clouds. One of the most popular methods is the Iterative Closest Point (ICP) algorithm developed by Besl and McKay ${ }^{1}$, Chen and Medioni ${ }^{2}$, and Zhang ${ }^{3}$. The ICP is based on the search of pairs of nearest points in the two sets, and estimating the rigid transformation, which aligns them. Then, the rigid transformation is applied to the points of one set, and the procedure is iterated until convergence. The ICP assumes that one point set is a subset of the other. When this assumption is not valid, false matches are created, that negatively influence the convergence of the ICP to the correct solution ${ }^{4}$.

Several variations and improvements of the ICP method have been made ${ }^{5,6}$. From a computational expense point of view it is highly time consuming due to the exhaustive search for the nearest point ${ }^{7}$. In Besl and McKay ${ }^{1}$, and Zhang's ${ }^{3}$ works the ICP requires every point in one surface to have a corresponding point on the other surface. An alternative approach to this search schema was proposed by Chen and Medioni ${ }^{2}$. They used the distance between the surfaces in the direction normal to the first surface as a registration evaluation function instead of point-to-nearest point distance. This point-totangent plane distance idea was originally proposed by Potmesil ${ }^{8}$. In Dorai et al. ${ }^{9}$ the method of Chen and Medioni was extended to an optimal weighted least-squares framework. Zhang ${ }^{3}$ proposed a thresholding technique using robust statistics to limit the maximum distance between points. Masuda and Yokoya ${ }^{5}$ used the ICP with random sampling and least median square error measurement that is robust to a partially overlapping scene. Okatani and Deguchi ${ }^{10}$ proposed the best transformation of two range images to align each other by taking into account the measurement error properties, which are mainly dependent on both the viewing direction and the distance to the object surface.

The ICP algorithm always converges monotonically to a local minimum with respect to the mean-square distance objective function $^{1}$. It does not use the local surface gradients in order to direct the solution to a minimum. Originally, it was not designed to register multi-scale range data. Several reviews and comparison studies about the ICP variant methods are available in the literature ${ }^{11-13}$. In Neugebauer ${ }^{14}$ and Szeliski and Lavallee ${ }^{15}$ two gradient descent type of algorithms were given. They adopt the Levenberg-Marquardt method for the estimation.

Since 3D point clouds derived by any method or device represent the object surface, the problem should be defined as a surface matching problem. In Photogrammetry, the problem statement of surface patch matching and its solution method was first addressed by Gruen ${ }^{16}$ as a straight extension of Least Squares Matching (LSM). 
There have been some studies on the absolute orientation of stereo models using Digital Elevation Models (DEM) as control information. This work is known as DEM matching. The absolute orientation of the models using Digital Terrain Models (DTM) as control information was first proposed by Ebner and Mueller ${ }^{17}$, and Ebner and Strunz ${ }^{18}$. Afterwards, the functional model of DEM matching has been formulated by Rosenholm and Torlegard ${ }^{19}$. This method basically estimates the 3D similarity transformation parameters between two DEM patches, minimizing the sum of squares of differences along the $Z$-axes. Several applications of DEM matching have been reported ${ }^{20-23}$.

Maas $^{24}$ successfully applied a similar method to register airborne laser scanner strips, among which vertical and horizontal discrepancies generally show up due to GPS/INS accuracy problems. Another similar method has been presented for registering surfaces acquired using different methods, in particular, laser altimetry and photogrammetry ${ }^{25}$. Furthermore, techniques for 2.5D DEM surface matching have been developed, which correspond mathematically to Least Squares Image Matching. The DEM matching concept can only be applied to 2.5D surfaces, whose analytic function is described in the explicit form as a single valued function, i.e. $z=f(x, y) .2 .5 \mathrm{D}$ surfaces are of limited value in case of generally formed objects.

When the surface curvature is either homogeneous or isotropic, as it is the case with all second order surfaces, e.g. plane or sphere, the geometry based registration techniques will probably fail. In some studies surface geometry and intensity (or color) information has been combined in order to solve this problem ${ }^{26-29}$.

The $\mathrm{LSM}^{16}$ concept has been applied to many different types of measurement and feature extraction problems due to its high level of flexibility and its powerful mathematical model: Adaptive Least Squares Image Matching, Geometrically Constrained Multiphoto Matching, Image Edge Matching, Multiple Patch Matching with 2D images, Multiple Cuboid (voxel) Matching with 3D images, Globally Enforced Least Squares Template Matching, Least Squares B-spline Snakes. For a detailed survey the authors refer to Gruen ${ }^{30}$. If 3D point clouds derived by any device or method represent an object surface, the problem should be defined as a surface matching problem instead of the point cloud matching. In particular, we treat it as least squares matching of overlapping 3D surfaces, which are digitized/sampled point by point using a laser scanner device, the photogrammetric method or other surface measurement techniques. This definition allows us to find a more general solution for the problem as well as to establish a flexible mathematical model in the context of LSM.

Our mathematical model is another generalization of the $\mathrm{LSM}^{16}$, as for the case of multiple cuboid matching in 3D voxel space $^{31,32}$.

Our proposed method, Least Squares 3D Surface Matching (LS3D), estimates the 3D transformation parameters between two or more fully 3D surface patches, minimizing the sum of squares of the Euclidean distances between the surfaces. This formulation gives the opportunity of matching arbitrarily oriented 3D surface patches. An observation equation is written for each surface element on the template surface patch, i.e. for each sampled point. The geometric relationship between the conjugate surface patches is defined as a 7-parameter 3D similarity transformation. This parameter space can be extended or reduced, as the situation demands it. The constant term of the adjustment is given by the observation vector whose elements are Euclidean distances between the template and search surface elements. Since the functional model is non-linear, the solution is iterative. The unknown transformation parameters are treated as stochastic quantities using proper a priori weights. This extension of the mathematical model gives control over the estimation parameters. The details of the mathematical modeling of the proposed method, precision and reliability issues, convergence behavior, and the computational aspects are explained in the following section. The further extensions to the basic model are given in the third section. Two practical examples for the demonstration of the basic method and the extensions are presented in the fourth section.

\section{LEAST SQUARES 3D SURFACE MATCHING (LS3D)}

\subsection{The basic estimation model}

Assume that two different partial surfaces of the same object are digitized/sampled point by point, at different times (temporally) or from different viewpoints (spatially). Although the conventional sampling pattern is point based, any other type of sampling pattern is also accepted. $f(x, y, z)$ and $g(x, y, z)$ are conjugate regions of the object in the left and right surfaces respectively. The problem statement is finding the correspondent part of the template surface patch $f(x, y, z)$ on the search surface $g(x, y, z)$.

$f(x, y, z)=g(x, y, z)$ 
According to Equation (1) each surface element on the template surface patch $f(x, y, z)$ has an exact correspondent surface element on the search surface $g(x, y, z)$, or vice-versa, if both of the surfaces would analytically be continuous surfaces without any deterministic or stochastic discrepancies. In order to model the stochastic discrepancies, which are assumed to be random errors, and may come from the sensor, environmental conditions or measurement method, a true error vector $e(x, y, z)$ is added as:

$$
f(x, y, z)-e(x, y, z)=g(x, y, z)
$$

Equation (2) are observation equations, which functionally relate the observations $f(x, y, z)$ to the parameters of $g(x, y, z)$. The matching is achieved by least squares minimization of a goal function, which represents the sum of squares of the Euclidean distances between the template and the search surface elements: $\sum\|d\|^{2}=$ min and in Gauss form $[d d]=\min$, where $d$ stands for the Euclidean distance. The final location is estimated with respect to an initial position of $g(x, y, z)$, the approximation of the conjugate search surface $g^{0}(x, y, z)$.

To express the geometric relationship between the conjugate surface patches, a 7-parameter 3D similarity transformation is used:

$$
\begin{aligned}
& x=t_{x}+m\left(r_{11} x_{0}+r_{12} y_{0}+r_{13} z_{0}\right) \\
& y=t_{y}+m\left(r_{21} x_{0}+r_{22} y_{0}+r_{23} z_{0}\right) \\
& z=t_{z}+m\left(r_{31} x_{0}+r_{32} y_{0}+r_{33} z_{0}\right)
\end{aligned}
$$

where $r_{i j}=\boldsymbol{R}(\omega, \varphi, \kappa)$ are the elements of the orthogonal rotation matrix, $\left[\begin{array}{lll}t_{x} & t_{y} & t_{z}\end{array}\right]^{\mathrm{T}}$ is the translation vector, and $m$ is the uniform scale factor.

Depending on the deformation between the template and the search surfaces, any other type of 3D transformations could be used, e.g. 12-parameter affine, 24-parameter tri-linear, or 30-parameter quadratic family of transformations.

In order to perform least squares estimation, Equation (2) must be linearized by Taylor expansion, ignoring $2^{\text {nd }}$ and higher order terms.

$$
f(x, y, z)-e(x, y, z)=g^{0}(x, y, z)+\frac{\partial g^{0}(x, y, z)}{\partial x} d x+\frac{\partial g^{0}(x, y, z)}{\partial y} d y+\frac{\partial g^{0}(x, y, z)}{\partial z} d z
$$

with

$d x=\frac{\partial x}{\partial p_{i}} d p_{i} \quad, \quad d y=\frac{\partial y}{\partial p_{i}} d p_{i} \quad, \quad d z=\frac{\partial z}{\partial p_{i}} d p_{i}$

where $p_{i} \in\left\{t_{x}, t_{y}, t_{z}, m, \omega, \varphi, \kappa\right\}$ is the $i$-th transformation parameter in Equation (3). Differentiation of Equation (3) gives:

$$
\begin{aligned}
& d x=d t_{x}+a_{10} d m+a_{11} d \omega+a_{12} d \varphi+a_{13} d \kappa \\
& d y=d t_{y}+a_{20} d m+a_{21} d \omega+a_{22} d \varphi+a_{23} d \kappa \\
& d z=d t_{z}+a_{30} d m+a_{31} d \omega+a_{32} d \varphi+a_{33} d \kappa
\end{aligned}
$$

where $a_{i j}$ are the coefficient terms, whose expansions are trivial. Using the following notation

$g_{x}=\frac{\partial g^{0}(x, y, z)}{\partial x} \quad, \quad g_{y}=\frac{\partial g^{0}(x, y, z)}{\partial y} \quad, \quad g_{z}=\frac{\partial g^{0}(x, y, z)}{\partial z}$

and substituting Equations (6), Equation (4) results in the following:

$$
\begin{array}{r}
-e(x, y, z)=g_{x} d t_{x}+g_{y} d t_{y}+g_{z} d t_{z}+\left(g_{x} a_{10}+g_{y} a_{20}+g_{z} a_{30}\right) d m+\left(g_{x} a_{11}+g_{y} a_{21}+g_{z} a_{31}\right) d \omega+ \\
\left(g_{x} a_{12}+g_{y} a_{22}+g_{z} a_{32}\right) d \varphi+\left(g_{x} a_{13}+g_{y} a_{23}+g_{z} a_{33}\right) d \kappa-\left(f(x, y, z)-g^{0}(x, y, z)\right)
\end{array}
$$

In the context of the Gauss-Markoff model, each observation is related to a linear combination of the parameters, which are variables of a deterministic unknown function. This function constitutes the functional model of the whole mathematical model. The terms $\left\{g_{x}, g_{y}, g_{z}\right\}$ are numeric $1^{\text {st }}$ derivatives of this function $g(x, y, z)$. 
Equation (8) gives in matrix notation

$-\boldsymbol{e}=\boldsymbol{A x}-\boldsymbol{l}, \boldsymbol{P}$

where $\boldsymbol{A}$ is the design matrix, $\boldsymbol{x}^{\mathrm{T}}=\left[\begin{array}{lll}d t_{x} & d t_{y} d t_{z} d m d \omega d \varphi d \kappa\end{array}\right]$ is the parameter vector, and $\boldsymbol{l}=f(x, y, z)-g^{0}(x, y, z)$ is the constant vector that consists of the Euclidean distances between the template and correspondent search surface elements. The template surface elements are approximated by the data points, on the other hand the search surface elements are represented in two different kind of piecewise forms (planar and bi-linear) optionally, which will be explained in the following. In general both surfaces can be represented in any kind of piecewise form.

With the statistical expectation operator $\mathrm{E}\{\}$ and the assumptions

$\boldsymbol{e} \sim \mathrm{N}\left(0, \sigma_{0}^{2} \boldsymbol{Q}_{l l}\right) \quad, \quad \sigma_{0}^{2} \boldsymbol{Q}_{l l}=\sigma_{0}^{2} \boldsymbol{P}_{l l}^{-1}=\boldsymbol{K}_{l l}=\mathrm{E}\left\{\boldsymbol{e} \boldsymbol{e}^{\mathrm{T}}\right\}$

the system (9) and (10) is a Gauss-Markoff estimation model. $\boldsymbol{Q}_{l l}, \boldsymbol{P}=\boldsymbol{P}_{l l}$ and $\boldsymbol{K}_{l l}$ stand for a priori cofactor, weight and covariance matrices respectively.

The unknown transformation parameters are treated as stochastic quantities using proper a priori weights. This extension gives advantages of control over the estimating parameters ${ }^{33}$. In the case of poor initial approximations for unknowns or badly distributed 3D points along the principal component axes of the surface, some of the unknowns, especially the scale factor $m$, may converge to a wrong solution, even if the scale factors between the surface patches are same. We introduce the additional observation equations on the system parameters as

$-\boldsymbol{e}_{b}=\boldsymbol{I} \boldsymbol{x}-\boldsymbol{l}_{b} \quad, \quad \boldsymbol{P}_{b}$

where $\boldsymbol{I}$ is the identity matrix, $\boldsymbol{l}_{b}$ is the (fictitious) observation vector for the system parameters, and $\boldsymbol{P}_{b}$ is the associated weight coefficient matrix. The weight matrix $\boldsymbol{P}_{b}$ has to be chosen appropriately, considering a priori information of the parameters. An infinite weight value $\left(\left(\boldsymbol{P}_{b}\right)_{i i} \rightarrow \infty\right)$ excludes the $i$-th parameter from the system, assigning it as constant, whereas zero weight $\left(\left(\boldsymbol{P}_{b}\right)_{i i}=0\right)$ allows the $i$-th parameter to vary freely, assigning it as unknown parameter in the classical meaning.

The least squares solution of the joint system Equations (9) and (11) gives as the Generalized Gauss-Markoff model the unbiased minimum variance estimation for the parameters

$$
\begin{aligned}
\hat{\boldsymbol{x}} & =\left(\boldsymbol{A}^{\mathrm{T}} \boldsymbol{P} \boldsymbol{A}+\boldsymbol{P}_{b}\right)^{-1}\left(\boldsymbol{A}^{\mathrm{T}} \boldsymbol{P} \boldsymbol{l}+\boldsymbol{P}_{b} \boldsymbol{l}_{b}\right) & & \text { solution vector } \\
\hat{\boldsymbol{\sigma}}_{0}^{2} & =\frac{\boldsymbol{v}^{\mathrm{T}} \boldsymbol{P} \boldsymbol{v}+\boldsymbol{v}_{b}^{\mathrm{T}} \boldsymbol{P}_{b} \boldsymbol{v}_{b}}{r} & & \text { variance factor } \\
\boldsymbol{v} & =\boldsymbol{A} \hat{\boldsymbol{x}}-\boldsymbol{l} & & \text { residual vector for surface observations } \\
\boldsymbol{v}_{b} & =\boldsymbol{I} \hat{\boldsymbol{x}}-\boldsymbol{l}_{b} & & \text { residual vector for parameter observations }
\end{aligned}
$$

where $^{\wedge}$ stands for the Least Squares Estimator, and $r$ is the redundancy. When the system converges, the solution vector converges to zero $(\hat{\boldsymbol{x}} \rightarrow 0)$. Then the residuals of the surface observations $(\boldsymbol{v})_{i}$ become the final Euclidean distances between the estimated search surface and the template surface patches.

$(v)_{i}=\hat{g}(x, y, z)_{i}-f(x, y, z)_{i} \quad, \quad i=\{1, \ldots, n\}$

The function values $g^{0}(x, y, z)$ in Equation (2) are actually stochastic quantities. This fact is neglected here to allow for the use of the Gauss-Markoff model and to avoid unnecessary complications, as typically done in LSM ${ }^{16}$. Since the functional model is non-linear, the solution is obtained iteratively. In the first iteration the initial approximations for the parameters must be provided: $p_{i}^{0} \in\left\{t_{x}^{0}, t_{y}^{0}, t_{z}^{0}, m^{0}, \omega^{0}, \varphi^{0}, \kappa^{0}\right\}$. After the solution vector (12) is solved, the search surface $g^{0}(x, y, z)$ is transformed to a new state using the updated set of transformation parameters, and the design matrix $\boldsymbol{A}$ and the constant vector $\boldsymbol{l}$ are re-evaluated. The iteration stops if each element of the alteration vector $\hat{\boldsymbol{x}}$ in Equation (12) falls below a certain limit:

$\left|d p_{i}\right|<c_{i} \quad, \quad d p_{i} \in\left\{d t_{x}, d t_{y}, d t_{z}, d m, d \omega, d \varphi, d \kappa\right\} \quad, \quad i=\{1,2, \ldots, 7\}$

The numerical derivative terms $\left\{g_{x}, g_{y}, g_{z}\right\}$ are defined as local surface normals. Their calculation depends on the analytical representation of the search surface elements. 
As a first method, let us represent the search surface elements as planar surface patches (Fig. 1a), which are constituted by fitting a plane to 3 neighboring knot points, in the non-parametric implicit form

$g^{0}(x, y, z)=A x+B y+C z+D=0$

where $A, B, C$, and $D$ are the parameters of the plane. The derivative terms are $x-y-z$ components of the local surface normal vector $\boldsymbol{n}$ at that point:

$\boldsymbol{n}=\left[\begin{array}{lll}g_{x} & g_{y} & g_{z}\end{array}\right]^{\mathrm{T}}=\frac{\left[\begin{array}{lll}A & B & C\end{array}\right]^{\mathrm{T}}}{\sqrt{A^{2}+B^{2}+C^{2}}}$

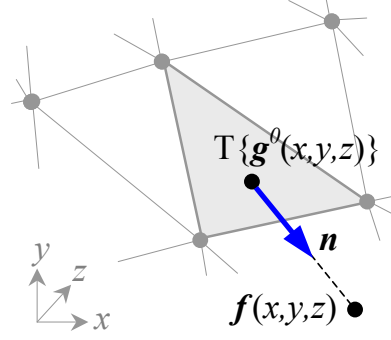

(a)

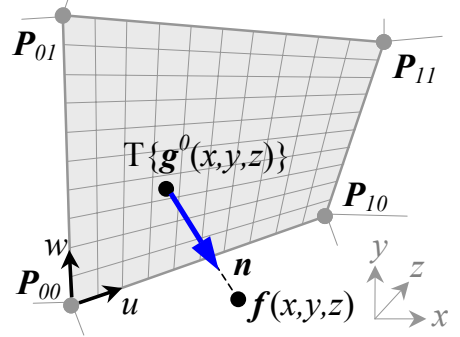

(b)
Fig. 1. Representation of surface elements in triangle plane form (a), and in parametric bi-linear form (b). Note that $\mathrm{T}\{\}$ stands for the transformation operator.

For the representation of the search surface elements as parametric bi-linear surface patches (Fig. 1-b), a bi-linear surface is fitted to 4 neighboring knot points $\boldsymbol{P}_{i j}$ :

$g^{0}(u, w)=\boldsymbol{P}_{00}(1-u)(1-w)+\boldsymbol{P}_{01}(1-u) w+\boldsymbol{P}_{10} u(1-w)+\boldsymbol{P}_{11} u w$

where $u, w \in[0,1]^{2}$ and $g^{0}(u, w), \boldsymbol{P}_{i j} \in \mathcal{R}^{3}$. The vector $g^{0}(u, w)$ is the position vector of any point on the bi-linear surface. Again the numeric derivative terms $\left\{g_{x}, g_{y}, g_{z}\right\}$ are calculated from components of the local surface normal vector $\boldsymbol{n}$ on the parametric bi-linear surface patch:

$\boldsymbol{n}=\left[\begin{array}{lll}g_{x} & g_{y} & g_{z}\end{array}\right]^{\mathrm{T}}=\frac{\partial g^{0}(u, w)}{\partial u} \times \frac{\partial g^{0}(u, w)}{\partial w} /\left\|\frac{\partial g^{0}(u, w)}{\partial u} \times \frac{\partial g^{0}(u, w)}{\partial w}\right\|$

where $\times$ stands for the vector cross product. With this approach a slightly better a posteriori sigma naught value could be obtained due to better surface modeling.

\subsection{Precision and reliability}

The standard deviations of the estimated transformation parameters and the correlations between themselves may give useful information concerning the stability of the system and quality of the data content ${ }^{16}$ :

$\hat{\boldsymbol{\sigma}}_{p}=\hat{\sigma}_{0} \sqrt{q_{p p}} \quad, \quad q_{p p} \in \boldsymbol{Q}_{x x}=\left(\boldsymbol{A}^{\mathrm{T}} \boldsymbol{P} \boldsymbol{A}+\boldsymbol{P}_{b}\right)^{-1}$

where $\boldsymbol{Q}_{x x}$ is the cofactor matrix for the estimated parameters. As pointed out in Maas ${ }^{24}$, the estimated standard deviations of the transformation parameters are usually too optimistic due to the stochastic properties of the search surface, which are not taken into consideration. In order to localize and eliminate the occluded parts and the outliers a simple weighting scheme adapted from the Robust Estimation Methods is used:

$(\boldsymbol{P})_{i i}= \begin{cases}1 & \text { if } \\ 0 & \text { else }\end{cases}$

In our experiments $K$ is selected as $>10$, since it is aimed to suppress only the large outliers. Because of the high redundancy of a typical data arrangement, a certain amount of occlusions and/or smaller outliers do not have significant effect on the estimated parameters. As a comprehensive strategy, Baarda's data-snooping method can be favourably used to localize the occluded or gross erroneous measurements.

\subsection{Convergence of solution vector}

In a standard least squares adjustment calculus, the function of the unknowns is unique, exactly known, and analytically continuous everywhere. Here the function $g(x, y, z)$ is discretized by using a finite sampling rate, which leads to slow convergence, oscillations, and even divergence in some cases with respect to the standard adjustment. The convergence behaviour of the proposed method basically depends on the quality of the initial approximations and quality of the data content. For a good data configuration it usually achieves the solution after 5 or 6 iterations (Fig. 2), which is typical for LSM. 


\subsection{Computational aspects}

The computational effort increases with the number of points in the matching process. The main portion of the computational complexity is to search the correspondent element of the template surface on the search surface patch, whereas the adjustment part is a small system, and can quickly be solved using Cholesky decomposition followed by back-substitution. Searching the correspondence is an algorithmic problem, and needs professional software optimization techniques and programming skills, which are not within the scope of this paper.

In the case of insufficient initial approximations, the numerical derivatives $\left\{g_{x}, g_{y}, g_{z}\right\}$ can also be calculated on the template surface patch $f(x, y, z)$ instead of on the search surface $g(x, y, z)$ in order to speed-up the convergence. This speed-up version apparently decreases the computational effort of the design matrix $\boldsymbol{A}$ as well, since the derivative terms $\left\{f_{x}, f_{y}, f_{z}\right\}$ are calculated only once in the first iteration, and the same values are used in the following iterations. As opposed to the basic model, the number of the observation equations contributing to the design matrix $\boldsymbol{A}$ is here defined by the number of elements on the search surface patch $g(x, y, z)$.

Two $1^{\text {st }}$ degree $C^{0}$ continuous surface representations are implemented. In the case of multi-resolution data sets, in which point densities are significantly different on the template and search surface patches, higher degree $\mathrm{C}^{1}$ continuous composite surface representations, e.g. bi-cubic Hermit surface ${ }^{34}$, should give better results, of course increasing the computational expenses.

\section{FURTHER EXTENSIONS TO THE BASIC MODEL}

\subsection{Simultaneous multi-subpatch matching}

The basic estimation model can be implemented in a multi-patch mode, that is the simultaneous matching of two or more search surfaces $g_{i}(x, y, z), i=1, \ldots, k$ to one template surface $f(x, y, z)$.

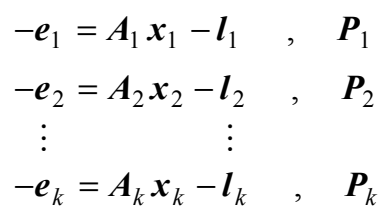

Since the parameter vectors $\boldsymbol{x}_{1}, \ldots, \boldsymbol{x}_{k}$ do not have any joint components, the sub-systems of Equation (24) are orthogonal to each other. In the presence of auxiliary information those sets of equations could be connected via functional constraints, e.g. as in the Geometrically Constrained Multiphoto Matching ${ }^{16,35}$ or via appropriate formulation of multiple $(>2)$ overlap conditions.

An ordinary point cloud includes enormously redundant information. A straightforward way to register such two point clouds could be matching of the whole overlapping areas. This is computationally expensive. We propose multi-subpatch mode as a further extension to the basic model, which is capable of simultaneous matching of sub-surface patches, which are selected in cooperative surface areas. They are joined to the system by the same 3D transformation parameters. This leads to the observation equations

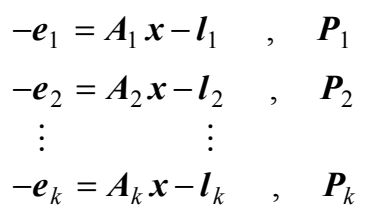

with $i=1, \ldots, k$ subpatches. They can be combined as in Equation (9), since the common parameter vector $\boldsymbol{x}$ joints them to each other. The individual subpatches may not include sufficient information for the matching of whole surfaces, but together they provide a computationally effective solution, since they consist of only relevant information rather than 
using the full data set. One must carefully select the distribution and size of the subpatches in order to get a homogeneous quality of the transformation parameters in all directions of the 3D space.

\subsection{Simultaneous matching of surface geometry and intensity}

In case of lack of sufficient geometric information (homogeneity or isotropicy of curvatures) the procedure may fail, since there is not a unique solution geometrically, e.g. in case of matching of two planes or spherical objects. An object surface may have some attribute information attached to it. Intensity, color, and temperature are well known examples. Most of the laser scanners can supply intensity information in addition to the Cartesian coordinates for each point, or an additional camera may be used to collect texture. We propose another further extension that can simultaneously match intensity information and geometry under a combined estimation model. In this approach the intensity image of the point cloud also contributes observation equations to the system, considering the intensities as supplementary information to the range image.

Rather than adopting a feature-based or step-wise approach our method sets up quasi-surfaces from intensity information in addition to the actual surfaces. A hypothetical example of forming the quasi-surfaces is given in Fig. 3. The procedure starts with the calculation of surface normal vectors at each data point. The actual surface will include noise and surface spikes (Fig. 3-b), which lead to unrealistic calculation for the normal vectors. To cope with the problem a moving average or median type filtering process could be employed. But still some noise would remain depending on the window size.

An optimum solution is the least squares fitting of a global trend surface to the whole point cloud (Fig. 3-c). It will suppress the noise component and preserves the global continuity of the normal vectors along the surface. We opt for the parametric bi-quadratic trend surface, which is sufficient to model the quadric type of surfaces, e.g. plane, sphere, ellipsoid, etc. For the template surface patch $f(x, y, z)$ we may write:

$$
\begin{aligned}
& F(u, w)=\sum_{i=0}^{2} \sum_{j=0}^{2} \boldsymbol{b}_{i j} u^{i} w^{j} \\
& F(u, w)=\boldsymbol{b}_{00}+\boldsymbol{b}_{01} w+\boldsymbol{b}_{02} w^{2}+\boldsymbol{b}_{10} u+\boldsymbol{b}_{11} u w+\boldsymbol{b}_{12} u w^{2}+\boldsymbol{b}_{20} u^{2}+\boldsymbol{b}_{21} u^{2} w+\boldsymbol{b}_{22} u^{2} w^{2}
\end{aligned}
$$

where $u, w \in[0,1]^{2}, F(u, w) \in \mathcal{R}^{3}$ is the position vector of any point on the trend surface, and $\boldsymbol{b}_{i j} \in \mathcal{R}^{3}$ are the algebraic coefficients, which are estimated by the least squares fitting. For each point the normal vector $\boldsymbol{n}_{f}$ is calculated on the trend surface $F(u, w)$ and attached to the actual surface $f(x, y, z)$ (Fig. 3-d):

$$
\boldsymbol{n}_{f}=\boldsymbol{n}_{f}(u, w)=\frac{\boldsymbol{F}_{u} \times \boldsymbol{F}_{w}}{\left\|\boldsymbol{F}_{u} \times \boldsymbol{F}_{w}\right\|}
$$

where $\boldsymbol{F}_{u}$ and $\boldsymbol{F}_{w}$ are the tangent vectors along the $u$ and $w$-axes respectively. Finally the quasi-surface $f_{c}(x, y, z)$ is formed in such a way that each point of the actual surface $f(x, y, z)$ is mapped along its normal vector $\boldsymbol{n}_{f}$ up to a distance proportional to its intensity value $c_{f}$ (Fig. 3-e).

$$
f_{c}(x, y, z)=f(x, y, z)+\boldsymbol{n}_{f} \lambda c_{f}
$$

where $\lambda$ is an appropriate scalar factor for the conversion from the intensity range to the Cartesian space. Rather than the actual surface $f(x, y, z)$ the trend surface $F(u, w)$ can also be defined as the datum, which leads to

$$
f_{c}(x, y, z)=F(u, w)+\boldsymbol{n}_{f} \lambda c_{f}
$$

This isolates the geometric noise component from the quasi-surface $f_{c}(x, y, z)$, but strongly smoothes the geometry. Equations (29) and (30) assume a fairly simplistic radiometric model (intensities are mapped perpendicular to the geometric surface). We will refine this model in subsequent work.

The same procedure is performed for the search surface $g(x, y, z)$ as well:

$$
g_{c}(x, y, z)=g(x, y, z)+\boldsymbol{n}_{g} \lambda c_{g}
$$

Equation (2) should also be valid for the quasi-surfaces under the assumption that similar illumination conditions exist for the both template and search surfaces: 


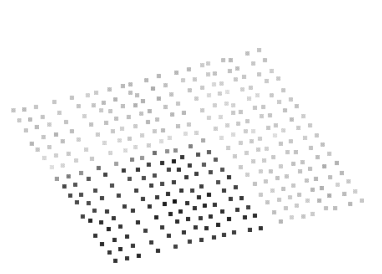

(a)

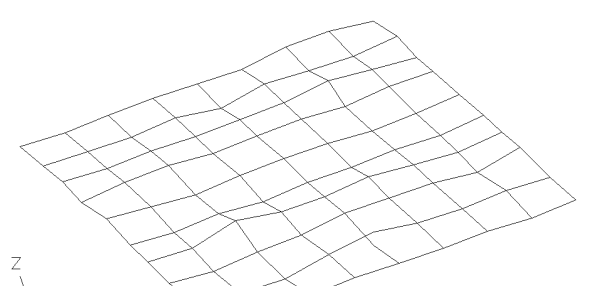

(b)

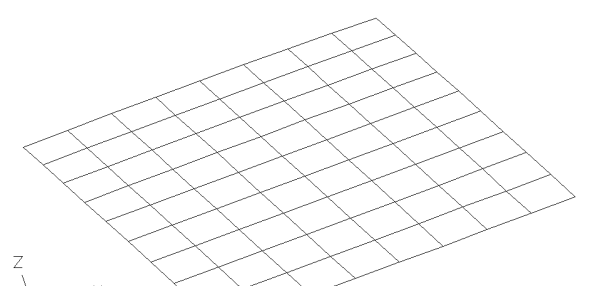

(c)
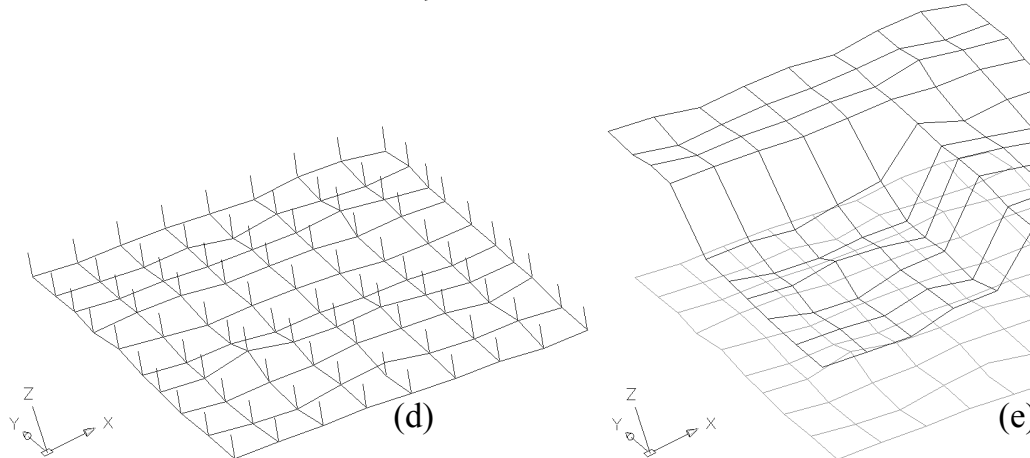

Fig. 3. Forming the quasi-surface. (a) Point cloud with intensity information, (b) meshed surface of the point cloud, (c) trend surface fitted to the point cloud, (d) noise-free normal vectors, (e) generated quasi-surface in addition to the actual one.

$$
f_{c}(x, y, z)-e_{c}(x, y, z)=g_{c}(x, y, z)
$$

The random errors of the template and search quasi-surfaces are assumed to be uncorrelated. The contrast and brightness differences or in the extreme case specular reflection will cause model errors, and deteriorate the reliability of the estimation. The radiometric variations between the template and search surface intensities should be adapted before matching by pre-processing or appropriate modeling in the estimation process by extra parameters.

For two images of an object acquired by an optical-passive sensor, e.g. a CCD camera, such an intensity transfer function $\left(c_{f}=t_{g}+c_{g} m_{g}\right)$ could be suitable for the radiometric adaptation, where $t_{g}$ (shift) and $m_{g}$ (scale) are radiometric correction parameters. In the case of laser scanner derived intensity images the radiometric variations are strongly dependent on both the incident angle of the signal path with respect to object surface normal and object-to-sensor distance. Then, for a plane type of object the radiometric variations can be modeled in first approximating as in the following:

$f_{c}(x, y, z)-e_{c}(x, y, z)=g_{c}(x, y, z)+t_{g}+u m_{g}$

where $u$ is the abscissa of the search trend surface $G(u, w)$, considering that $u$-axis is the horizontal direction. In other words, $u$-axis is the principal direction of changing of the incident angles. Depending on the characteristics of scan data it can be replaced by ordinate value $w$, or another type of parameterization. Although the radiometric parameters $t_{g}$ and $m_{g}$ are linear a priori, we expand them to Taylor series. Equation (33) in linearized form gives:

$-e_{c}(x, y, z)=g_{c x} d x+g_{c y} d y+g_{c z} d z+d t_{g}+u d m_{g}-\left(f_{c}(x, y, z)-\left(g_{c}^{0}(x, y, z)+\left\{t_{g}^{0}+u m_{g}^{0} ; \boldsymbol{n}_{g}\right\}\right)\right)$

where $g_{c x}, g_{c y}$, and $g_{c z}$ stand for the derivative terms like as given in Equation (7) for the actual surface observations. The first approximations of the radiometric parameters are $t_{g}{ }^{0}=m_{g}{ }^{0}=0$. At the end of the each iteration the quasi search surface $g_{c}^{0}(x, y, z)$ is transformed to a new state using the updated set of transformation parameters, and subsequently reshaped by the current set of radiometric parameters $t_{g}{ }^{0}+u m_{g}{ }^{0}$ along the normal vectors $\boldsymbol{n}_{g}$, which are calculated on the search trend surface $G(u, w)$.

The quasi-surfaces are treated like actual surfaces in the estimation model. They contribute observation equations to the design matrix, joining the system by the same set of transformation parameters. After the further expansion of Equation (34) and with the assumptions $\mathrm{E}\left\{\boldsymbol{e}_{c}\right\}=0$ and $\mathrm{E}\left\{\boldsymbol{e}_{c} \boldsymbol{e}_{c}^{\mathrm{T}}\right\}=\sigma_{0}{ }^{2} \boldsymbol{P}_{c}^{-1}$, the total system becomes

$$
\begin{array}{lll}
-\boldsymbol{e}=\boldsymbol{A} \boldsymbol{x}-\boldsymbol{l} & , & \boldsymbol{P} \\
-\boldsymbol{e}_{b}=\boldsymbol{I} \boldsymbol{x}-\boldsymbol{l}_{b} & , & \boldsymbol{P}_{b} \\
-\boldsymbol{e}_{c}=\boldsymbol{A}_{c} \boldsymbol{x}-\boldsymbol{l}_{c} & , & \boldsymbol{P}_{c}
\end{array}
$$


where $\boldsymbol{e}_{c}, \boldsymbol{A}_{c}$, and $\boldsymbol{P}_{c}$ are the true error vector, the design matrix, and the associated weight coefficient matrix for the quasi-surface observations respectively, and $\boldsymbol{l}_{c}$ is the constant vector that contains the Euclidean distances between the template and correspondent search quasi-surfaces elements. The hybrid system in Equation (35) is of the combined adjustment type that allows simultaneous matching of geometry and intensity.

In our experiments, weights for the quasi-surface observations are selected as $\left(\boldsymbol{P}_{c}\right)_{i i}<(\boldsymbol{P})_{i i}$, and the intensity measurements of the (laser) sensor are considered to be uncorrelated with the distance measurements $\left(E\left\{\boldsymbol{e}_{c} \boldsymbol{e}^{\mathrm{T}}\right\}=0\right)$ for the sake of simplicity of the stochastic model.

\section{EXPERIMENTAL RESULTS}

Two practical examples are given to show the capabilities of the method. All experiments were carried out using own self-developed $\mathrm{C} / \mathrm{C}++$ software that runs on Microsoft Windows ${ }^{\circledR}$ OS. In all experiments the initial approximations of the unknowns were provided by interactively selecting 3 common points on both surfaces before matching. Since in all data sets there was no scale difference, the scale factor $m$ was fixed to unity by infinite weight value $\left(\left(\boldsymbol{P}_{b}\right)_{i i} \rightarrow \infty\right)$. The iteration criteria values $c_{i}$ were selected as $1.0 \mathrm{e}-4$ meters for the elements of the translation vector and $1.0 \mathrm{e}-3$ gon for the rotation angles.

The first example is the registration of three point clouds of a plant (Fig. 4). The scanning was performed by the HDS 2500 (Leica Geosystems) laser scanner. The average point spacing is 12 millimeters. The point clouds Fig. 4-a and 4-c were matched to Fig. 4-b by use of the LS3D surface matching. In this experiment the whole overlapping areas were matched. The numerical results of the matching of the first and third point clouds are given at parts I and II of Table 1 respectively. Even though it is a very complex environment, the matching process is quite successful. Relatively homogeneous and small magnitudes of the theoretical precisions of the parameters show an excellent fit along all directions. However the theoretical precisions are too optimistic.
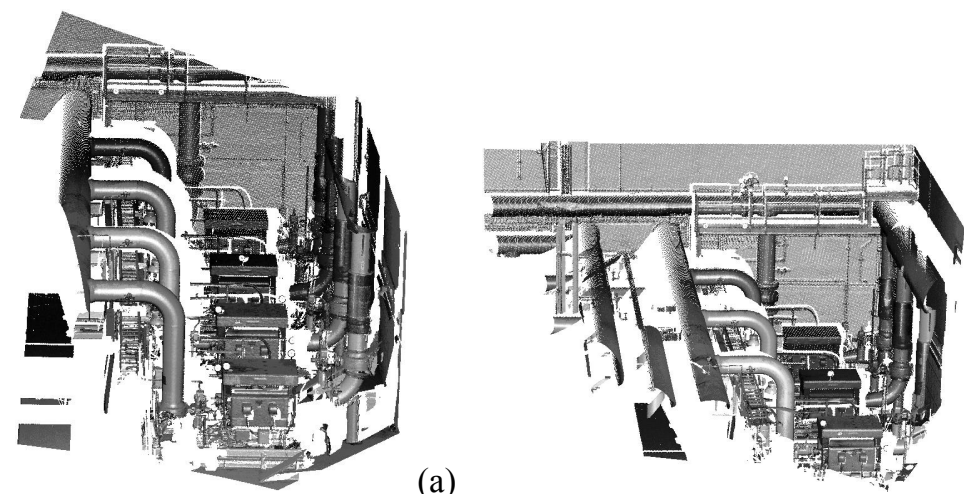

(b)
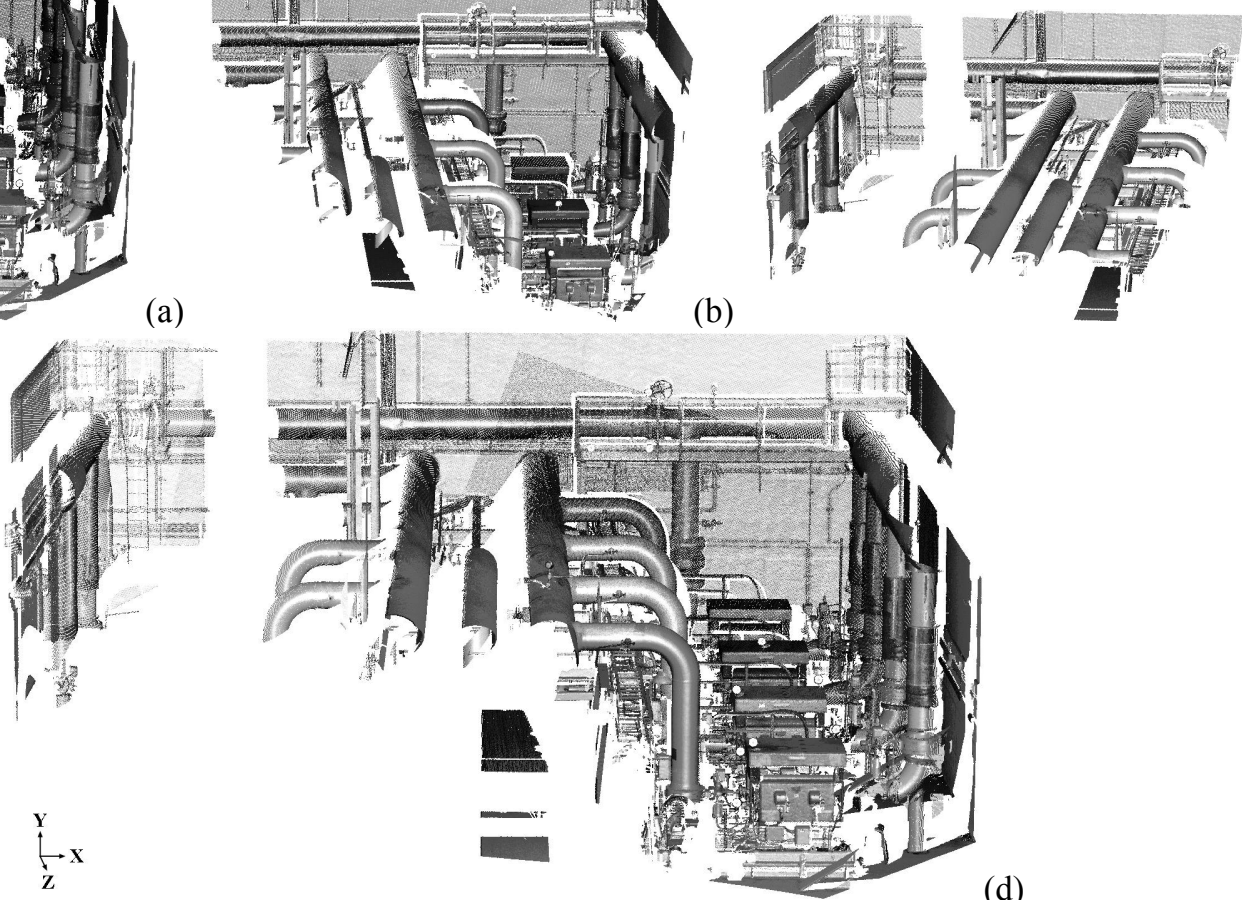

(c)

Fig. 4. Example "plant". (a), (b), (c) First, second and third point cloud, (d) composite point cloud after the LS3D surface matching. Note that laser scanner derived intensities are back-projected onto the point clouds. 
Table 1: Numerical results of "plant" example

\begin{tabular}{lcccccc}
\hline$\#$ & $\begin{array}{c}\text { surf. } \\
\text { mode }\end{array}$ & $\sum$ points & Iter. & $\begin{array}{c}\hat{\sigma}_{0} \\
(\mathrm{~mm})\end{array}$ & $\begin{array}{c}\hat{\sigma}_{t x} / \hat{\sigma}_{t y} / \hat{\sigma}_{t z} \\
(\mathrm{~mm})\end{array}$ & $\begin{array}{c}\hat{\sigma}_{\omega} / \hat{\sigma}_{\varphi} / \hat{\sigma}_{\kappa} \\
(1.0 \mathrm{e}-02 \text { gon })\end{array}$ \\
\hline $\mathrm{I}$ & $\mathrm{P}$ & 245041 & 6 & 2.78 & $0.03 / 0.03 / 0.01$ & $0.01 / 0.01 / 0.03$ \\
& $\mathrm{~B}$ & & 5 & 2.79 & $0.03 / 0.03 / 0.01$ & $0.01 / 0.01 / 0.03$ \\
\hline $\mathrm{II}$ & $\mathrm{P}$ & 323936 & 7 & 2.54 & $0.02 / 0.02 / 0.01$ & $0.01 / 0.01 / 0.02$ \\
& $\mathrm{~B}$ & & 6 & 2.52 & $0.02 / 0.02 / 0.01$ & $0.01 / 0.01 / 0.02$ \\
\hline $\mathrm{III}$ & $\mathrm{P}$ & 20407 & 6 & 2.11 & $0.09 / 0.09 / 0.04$ & $0.05 / 0.04 / 0.08$ \\
& $\mathrm{~B}$ & & 5 & 2.09 & $0.09 / 0.09 / 0.04$ & $0.05 / 0.04 / 0.08$ \\
\hline $\mathrm{IV}$ & $\mathrm{P}$ & 37983 & 8 & 2.01 & $0.04 / 0.04 / 0.02$ & $0.03 / 0.03 / 0.07$ \\
& $\mathrm{~B}$ & & 8 & 2.00 & $0.04 / 0.05 / 0.02$ & $0.03 / 0.03 / 0.07$ \\
\hline
\end{tabular}

P: plane surface representation, B: bi-linear surface representation
A further matching process was carried out using the simultaneous multi-subpatch approach of the LS3D. For the first point cloud 5 and for the third point cloud 7 occlusion-free cooperative subpatches were selected. The results of the matching of the first and third point clouds are given at parts III and IV of Table 1 respectively. Although the precision values increase while the system redundancy decreases, they are still optimistic mainly due to the stochastic properties of the search surfaces.

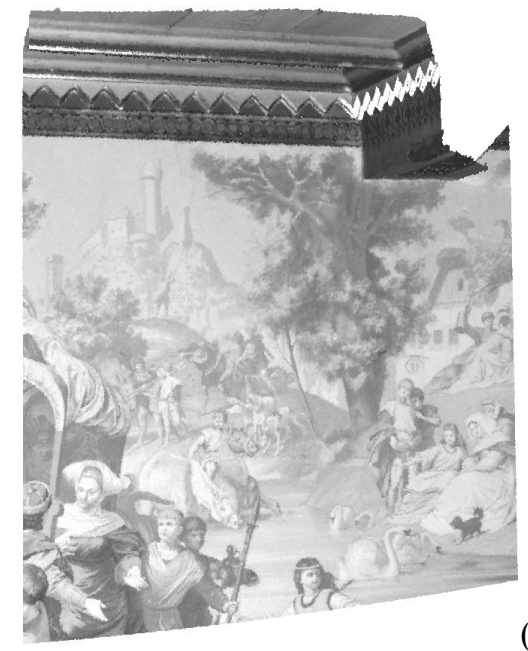

(a)
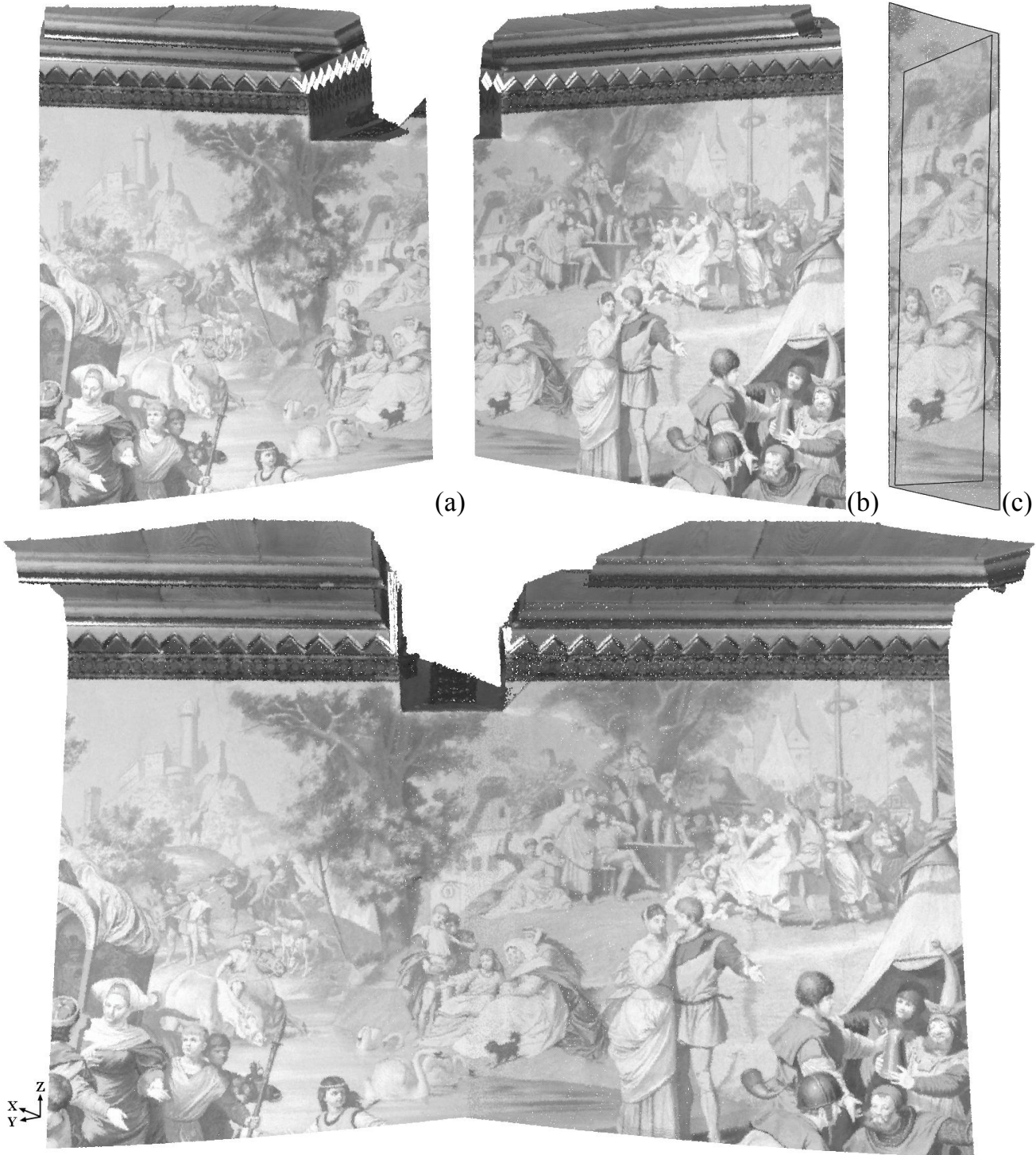

(d)

Fig. 5. Example "Neuschwanstein". (a) Left point cloud, (b) right point cloud, (c) coverage of the template patch (inner quadrangle) and the search patch (outer quadrangle), which are selected on the left and right point clouds respectively, (d) composite point cloud after the simultaneous matching of geometry and intensity by LS3D. Note that laser scanner derived intensities are back-projected onto the point clouds. 
The second experiment refers to simultaneous matching of surface geometry and intensity. Two scans of a wall painting in Neuschwanstein Castle in Bavaria, Germany, were matched (Fig. 5). The scanning was performed using the IMAGER 5003 (Zoller+Fröhlich) laser scanner. The average point spacing is 3 millimeters. Laser scanner derived reflectance values were used as intensity information. The actual surface observations are considered as the unit weight $(\boldsymbol{P})_{i i}=1$. Consequently weights for the quasi-surface observations are selected as $\left(\boldsymbol{P}_{c}\right)_{i i}=0.75$. The iteration criteria values $c_{i}$ were selected as $2.0 \mathrm{e}-4$ meters for the elements of the translation vector and $5.0 \mathrm{e}-3$ gon for the rotation angles. The search surface, selected on the right point cloud, was matched to the template one, which was selected on the left point cloud (Fig. 5-c). The numerical results are given in Table 2.

Table 2: Numerical results of "Neuschwanstein" example

\begin{tabular}{lccccccc}
\hline$\#$ & $\begin{array}{c}\text { surf. } \\
\text { mode }\end{array}$ & datum Epoints & Iter. & $\begin{array}{c}\text { RMSE } \\
(\mathrm{mm})\end{array}$ & $\begin{array}{c}\hat{\sigma}_{t x} / \hat{\sigma}_{t y} / \hat{\sigma}_{t z} \\
(\mathrm{~mm})\end{array}$ & $\begin{array}{c}\hat{\sigma}_{\omega} / \hat{\sigma}_{\varphi} / \hat{\sigma}_{\kappa} \\
(1.0 \mathrm{e}-02 \text { gon })\end{array}$ \\
\hline $\mathrm{V}$ & $\mathrm{P}$ & $\mathrm{f} / \mathrm{g}$ & 45652 & 14 & 1.16 & $0.26 / 0.06 / 0.07$ & $0.15 / 0.16 / 0.53$ \\
& $\mathrm{~B}$ & $\mathrm{f} / \mathrm{g}$ & & 12 & 1.18 & $0.26 / 0.06 / 0.07$ & $0.15 / 0.16 / 0.53$ \\
\hline $\mathrm{VI}$ & $\mathrm{P}$ & $\mathrm{F} / \mathrm{G}$ & 45652 & 12 & 1.16 & $0.25 / 0.06 / 0.07$ & $0.15 / 0.15 / 0.50$ \\
& $\mathrm{~B}$ & $\mathrm{~F} / \mathrm{G}$ & & 12 & 1.18 & $0.25 / 0.06 / 0.07$ & $0.15 / 0.15 / 0.50$ \\
\hline
\end{tabular}

$\mathrm{f} / \mathrm{g}$ : datum are the actual surfaces $f / g(x, y, z), \mathrm{F} / \mathrm{G}$ : datum are the trend surfaces $F / G(u, w)$ RMSE: root mean square error of the residuals of the actual surface observations
Since the object is a plane, only surface geometry is not enough for the matching. Using the combined matching of surface geometry and intensity approach of the LS3D a successful solution has been achieved. The datum as the trend surface option gives a better convergence rate.

\section{CONCLUSIONS}

An algorithm for the least squares matching of overlapping 3D surfaces is presented. Our proposed method, the Least Squares 3D Surface Matching (LS3D), estimates the transformation parameters between two or more fully 3D surfaces, using the Generalized Gauss Markoff model, minimizing the sum of squares of the Euclidean distances between the surfaces. The mathematical model is a generalization of the least squares image matching method and offers high flexibility for any kind of 3D surface correspondence problem. The least squares concept allows for the monitoring of the quality of the final results by means of precision and reliability criterions. By appropriately selecting the 3D transformation method and the surface representation type, it is able to match multi-resolution, multi-temporal, multiscale, and multi-sensor data sets.

The capabilities of the technique are illustrated by a practical example. There are several ways to extend the technique. Here we give two of them, which are simultaneous matching of surface geometry and intensity under a combined estimation model and simultaneous multi-subpatch matching. Future studies will include more practical examples to demonstrate the full power of the technique.

The technique can be applied to a great variety of data co-registration problems. Since it reveals the sensor noise level and accuracy potential of any kind of surface measurement method or device, it can be used for comparison and validation studies. In addition time dependent (temporal) variations of the object surface can be inspected, tracked, and localized using the statistical analysis tools of the method.

\section{ACKNOWLEDGEMENTS}

The laser scanner data set "plant" is courtesy of Leica Geosystems HDS Inc., and data set "Neuschwanstein" is courtesy of Zoller+Fröhlich GmbH Elektrotechnik. The first author is financially supported by an ETH-Zurich Internal Research Grant, which is gratefully acknowledged.

\section{REFERENCES}

1. P.J. Besl, N.D. McKay, A method for registration of 3D shapes. IEEE Trans. Pattern Anal. Mach. Intel., 14(2), 239-256, 1992.

2. Y. Chen, G. Medioni, Object modelling by registration of multiple range images. Img. and Vis. Comp., 10(3), 145-155, 1992.

3. Z. Zhang, Iterative point matching for registration of free-form curves and surfaces. Int. J. of Comp. Vis., 13(2), 119-152, 1994.

4. A. Fusiello, U. Castellani, L. Ronchetti, V. Murino, Model acquisition by registration of multiple acoustic range views. Computer Vision - ECCV 2002, LNCS, vol. 2351, Springer, 805-819, 2002.

5. T. Masuda, N. Yokoya, A robust method for registration and segmentation of multiple range images. Comp. Vis. and Img. Under., 61(3), 295-307, 1995. 
6. R. Bergevin, M. Soucy, H. Gagnon, D. Laurendeau, Towards a general multi-view registration technique. IEEE Trans. Pattern Anal. Mach. Intel., 18(5), 540-547, 1996.

7. V. Sequeira, K. Ng, E. Wolfart, J.G.M. Goncalves, D. Hogg, Automated reconstruction of $3 D$ models from real environments. ISPRS J. of Photog. \& Rem. Sensing, 54(1), 1-22, 1999.

8. M. Potmesil, Generating models of solid objects by matching $3 D$ surface segments. Int. Joint Conf. on Artificial Intelligence, Karlsruhe, 1089-1093, 1983.

9. C. Dorai, J. Weng, A.K. Jain, Optimal registration of object views using range data. IEEE Trans. Pattern Anal. Mach. Intel., 19(10), 1131-1138, 1997.

10. I.S. Okatani, K. Deguchi, A method for fine registration of multiple view range images considering the measurement error properties. Comp. Vis. and Img. Under., 87(1-3), 66-77, 2002.

11. O. Jokinen, H. Haggren, Statistical analysis of two 3-D registration and modeling strategies. ISPRS J. of Photog. \& Rem. Sensing, 53(6), 320-341, 1998.

12. J.A. Williams, M. Bennamoun, S. Latham, Multiple view 3D registration: A review and a new technique. IEEE Int. Conf. Systems, Man, and Cybernetics, Tokyo, 497-502, 1999.

13. R.J. Campbell, P.J. Flynn, A survey of free-form object representation and recognition techniques. Comp. Vis. and Img. Under., 81(2), 166-210, 2001.

14. P.J. Neugebauer, Reconstruction of real-world objects via simultaneous registration and robust combination of multiple range images. Int. J. of Shape Modeling, 3(1-2), 71-90, 1997.

15. R. Szeliski, S. Lavallee, Matching 3-D anatomical surfaces with non-rigid deformations using octree-splines. Int. J. of Comp. Vis., 18(2), 171-186, 1996

16. A. Gruen, Adaptive least squares correlation: a powerful image matching technique. South Afr. J. of Photog., Rem. Sensing and Cartography, 14(3), 175-187, 1985.

17. H. Ebner, F. Mueller, Processing of Digital Three Line Imagery using a generalized model for combined point determination. Int. Arch. of Photog. and Rem. Sensing, 26(3/1), 212-222, 1986.

18. H. Ebner, G. Strunz, Combined point determination using Digital Terrain Models as control information. Int. Arch. of Photog. and Rem. Sensing, 27(B11/3), 578-587, 1988.

19. D. Rosenholm, K. Torlegard, Three-dimensional absolute orientation of stereo models using Digital Elevation Models. Photog. Eng. \& Rem. Sensing, 54(10), 1385-1389, 1988.

20. G.E. Karras, E. Petsa, DEM matching and detection of deformation in close-range Photogrammetry without control. Photog. Eng. \& Rem. Sensing, 59(9), 1419-1424, 1993.

21. L. Pilgrim, Robust estimation applied to surface matching. ISPRS J. of Photog. \& Rem. Sensing, 51(5), 243-257, 1996.

22. H.L. Mitchell, R.G. Chadwick, Digital Photogrammetric concepts applied to surface deformation studies. Geomatica, 53(4), 405414, 1999.

23. Z. Xu, Z. Li, Least median of squares matching for automated detection of surface deformations. Int. Arch. of Photog. and Rem. Sensing, 33(B3), 1000-1007, 2000.

24. H.G. Maas, Least-Squares Matching with airborne laserscanning data in a TIN structure. Int. Arch. of Photog. and Rem. Sensing, 33(3A), 548-555, 2000

25. Y. Postolov, A. Krupnik, K. McIntosh, Registration of airborne laser data to surfaces generated by Photogrammetric means. Int. Arch. of Photog. and Rem. Sensing, 32(3/W14), 95-99, 1999.

26. S. Weik, Registration of 3-D partial surface models using luminance and depth information. IEEE Int. Conf. 3D Digital Imaging and Modeling, Ottawa, 93-100, 1997.

27. A.E. Johnson, S.B. Kang, Registration and integration of textured 3D data. Img. and Vis. Comp., 17(2), 135-147, 1999.

28. H.G. Maas, On the use of pulse reflectance data for laserscanner strip adjustment. Int. Arch. of Photog. and Rem. Sensing, 34(3/W4), 2001.

29. J. Vanden Wyngaerd, L. Van Gool, Combining texture and shape for automatic crude patch registration. IEEE Int. Conf. 3D Digital Imaging and Modeling, Banff, 179-186, 2003.

30. A. Gruen, Least squares matching: a fundamental measurement algorithm. In: K. Atkinson (ed.), Close Range Photogrammetry and Machine Vision, Whittles, 217-255, 1996.

31. H.G. Maas, A high-speed solid state camera system for the acquisition of flow tomography sequences for $3 D$ least squares matching. Int. Arch. of Photog. and Rem. Sensing, 30(5), 241-249, 1994.

32. H.G. Maas, A. Gruen, Digital photogrammetric techniques for high resolution three dimensional flow velocity measurements. Opt. Eng., 34(7), 1970-1976, 1995.

33. A. Gruen, Photogrammetrische Punktbestimmung mit der Buendelmethode. IGP ETH Zurich, Mitteilungen Nr.40, 1986.

34. G.J. Peters, Interactive computer graphics application of the parametric bi-cubic surface to engineering design problems. In: Computer Aided Geometric Design, Academic Press, 259-302, 1974.

35. A. Gruen, E.P. Baltsavias, Geometrically Constrained Multiphoto Matching. Photog. Eng. \& Rem. Sensing, 54(5), 633-641, 1988. 